\section{A multipurpose four-dimensional analysis of variance program*}

\author{
RICHARD A. GALOSY \\ Hostos College, City University of New York, Bronx, New York 10451 \\ and \\ WILLIAM A. ADAMS \\ University of Wisconsin, Milwaukee, Wisconsin 53201
}

A computer program is described for a four-dimensional analysis of variance using the IBM $360 / 40$ OS, or larger, machine.

Behavioral scientists often find themselves designing experiments which necessitate an extension of available statistical procedures and result in computations which are beyond the scope of the hand calculator. In addition, these extended designs are often not found in published programs such as those of Veldman (1967).

The present program was written, tested, and utilized for the statistical analysis necessary for a comparative study (Lippman, Galosy, \& Thompson, 1970) which investigated the effects of three variables (handling, training, and species) over a series of repeated measures (days).

The statistical design is an extension of Lindquist's (1953) Type III ANOVA analysis of variance design, and of the computational procedures of Bruning and Kintz (1968). The extensions involve the addition of one between, or independent, comparison to the standard three-factor analysis, resulting in a four-dimensional ANOVA with three between comparisons and one within, or correlated, comparison. Because of the computational similarities of the four-dimensional, Type III, and Type I ANOVA (Bruning \& Kintz, 1968; Lindquist, 1953), it was subsequently amended to perform any of the three ANOVAs.

\section{PROGRAM CHARACTERISTICS}

Programming Language

$P L / I$ is the programming language.

\section{Type of Hardware}

IBM $360 / 40$ OS, or larger, should be used.

\section{Processing Performed}

The program will perform, at the user's option, any one of the following ANOVAs: two-factor, repeated

* Requests for reprints and test data should be sent to Richard A. Galosy, Department of Psychology: Developmental, University of North Carolina, Chapel Hill, N.C. 27514. measures on one; three-factor, repeated measures on one; and four-factor, repeated measures on one.

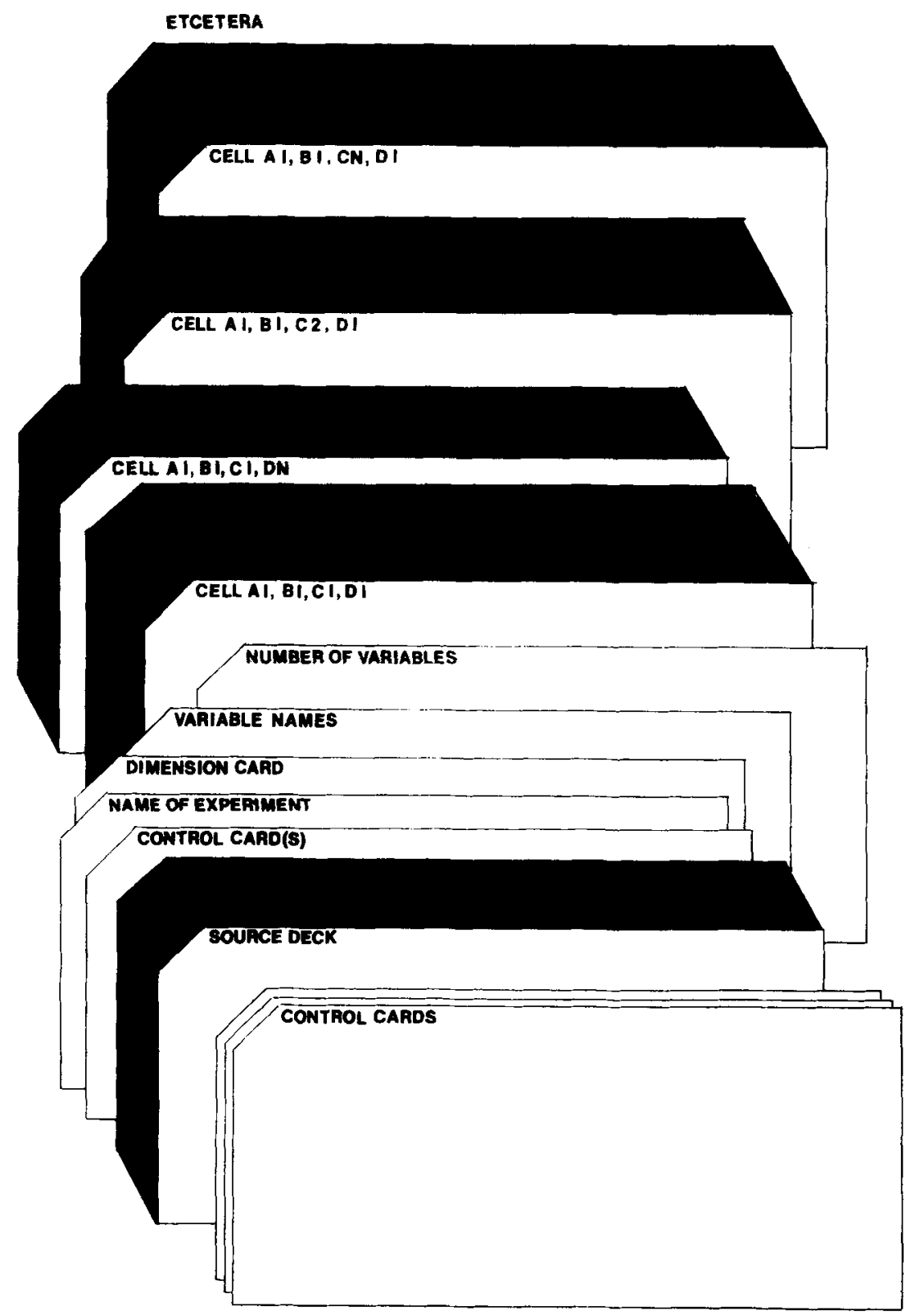

Fig. 1. A three-dimensional representation of the card deck before submitting the program for a run.
The program computes the mean squares, degrees of freedom, F ratios, and the probability of each F ratio. Individual cell means and standard deviations are also computed.

\section{Storage Requirements}

In most cases, $96 \mathrm{~K}$ has been sufficient. However, if more than approximately $100 \mathrm{Ss}$ are used, or more than approximately seven repeated measures are taken, auxiliary storage may be necessary.

Time

With about 30 Ss in a 2 by 2 by 2 by 4 four-dimensional analysis, the total job time is about $5 \mathrm{~min}$. The smaller designs take less time 
Table 1

Summary Table With Cell Means and Standard Deviations for the Type 34 ANOVA Using Test Data

\begin{tabular}{|c|c|c|c|c|c|}
\hline $\begin{array}{c}\text { This is a Test Run } \\
\text { Source }\end{array}$ & SS & DF & MS & $\mathbf{F}$ & $\mathbf{P}$ \\
\hline Total & 659.83 & 95.00 & & & \\
\hline $\begin{array}{l}\text { Between Ss } \\
\text { A (Handling) } \\
\text { B (Species) } \\
\text { C (Training) } \\
\text { A by B } \\
\text { A by C } \\
\text { B by C } \\
\text { A by B by C } \\
\text { Error Bet }\end{array}$ & $\begin{array}{r}274.33 \\
0.67 \\
5.04 \\
13.50 \\
15.04 \\
16.67 \\
51.04 \\
9.38 \\
163.00\end{array}$ & $\begin{array}{r}23.00 \\
1.00 \\
1.00 \\
1.00 \\
1.00 \\
1.00 \\
1.00 \\
1.00 \\
16.00\end{array}$ & $\begin{array}{r}0.67 \\
5.04 \\
13.50 \\
15.04 \\
16.67 \\
51.04 \\
9.38 \\
10.19\end{array}$ & $\begin{array}{l}0.07 \\
0.49 \\
1.33 \\
1.48 \\
1.64 \\
5.07 \\
0.92\end{array}$ & $\begin{array}{l}0.85739 \\
0.50182 \\
0.26595 \\
0.24063 \\
0.21722 \\
0.03780 \\
0.64813\end{array}$ \\
\hline $\begin{array}{l}\text { Within Ss } \\
\text { D (Days) } \\
\text { A by D } \\
\text { B by D } \\
\text { C by D } \\
\text { A by B by D } \\
\text { A by C by D } \\
\text { B by C by D } \\
\text { A by B by C by D } \\
\text { Error Win }\end{array}$ & \begin{tabular}{r|}
385.50 \\
3.42 \\
1.08 \\
49.04 \\
75.42 \\
40.21 \\
47.08 \\
19.04 \\
31.21 \\
119.00
\end{tabular} & $\begin{array}{r}72.00 \\
3.00 \\
3.00 \\
3.00 \\
3.00 \\
3.00 \\
3.00 \\
3.00 \\
3.00 \\
48.00\end{array}$ & $\begin{array}{r}1.14 \\
0.36 \\
16.35 \\
25.14 \\
13.40 \\
15.69 \\
6.35 \\
10.40 \\
2.48\end{array}$ & $\begin{array}{r}0.46 \\
0.15 \\
6.59 \\
10.14 \\
5.41 \\
6.33 \\
2.56 \\
4.20\end{array}$ & $\begin{array}{l}0.71637 \\
0.95227 \\
0.00109 \\
0.00010 \\
0.00309 \\
0.00136 \\
0.06481 \\
0.01030\end{array}$ \\
\hline
\end{tabular}

\begin{tabular}{|c|c|c|c|c|c|}
\hline \multicolumn{4}{|c|}{ Cell } & \multirow[b]{2}{*}{ Mean } & \multirow[b]{2}{*}{ SD } \\
\hline A & B & C & $\mathrm{D}$ & & \\
\hline 1 & 1 & 1 & 1 & 4.00 & 1.000 \\
\hline 1 & 1 & 1 & 2 & 3.67 & 2.082 \\
\hline 1 & 1 & 1 & 3 & 5.67 & 1.528 \\
\hline 1 & 1 & 1 & 4 & 6.00 & 1.000 \\
\hline 1 & 1 & 2 & 1 & 9.00 & 1.000 \\
\hline 1 & 1 & 2 & 2 & 9.00 & 1.000 \\
\hline 1 & 1 & 2 & 3 & 4.00 & 2.000 \\
\hline 1 & 1 & 2 & 4 & 5.33 & 2.082 \\
\hline 1 & 2 & 1 & 1 & 4.00 & 3.000 \\
\hline 1 & 2 & 1 & 2 & 5.33 & 3.055 \\
\hline 1 & 2 & 1 & 3 & 7.33 & 1.528 \\
\hline 1 & 2 & 1 & 4 & 6.00 & 3.606 \\
\hline 1 & 2 & 2 & 1 & 3.67 & 1.528 \\
\hline 1 & 2 & 2 & 2 & 4.00 & 2.646 \\
\hline 1 & 2 & 2 & $\mathbf{3}$ & 4.67 & 2.082 \\
\hline 1 & 2 & 2 & 4 & 1.67 & 0.577 \\
\hline 2 & 1 & 1 & 1 & 6.67 & 3.512 \\
\hline 2 & 1 & 1 & 2 & 2.00 & 1.000 \\
\hline 2 & 1 & 1 & 3 & 4.00 & 2.000 \\
\hline 2 & 1 & 1 & 4 & 3.33 & 1.528 \\
\hline 2 & 1 & 2 & 1 & 6.67 & 3.512 \\
\hline 2 & 1 & 2 & 2 & 8.67 & 0.577 \\
\hline 2 & 1 & 2 & $\mathbf{3}$ & 7.00 & 1.000 \\
\hline 2 & 1 & $\mathbf{2}$ & 4 & 3.33 & 1.528 \\
\hline 2 & 2 & 1 & $i$ & 4.67 & 2.082 \\
\hline 2 & 2 & 1 & 2 & 3.67 & 2.887 \\
\hline 2 & 2 & 1 & 3 & 7.00 & 2.000 \\
\hline 2 & 2 & 1 & 4 & 5.33 & 3.215 \\
\hline 2 & 2 & 2 & 1 & 3.33 & 1.528 \\
\hline 2 & 2 & 2 & 2 & 7.33 & 1.528 \\
\hline 2 & 2 & 2 & 3 & 4.00 & 2.646 \\
\hline 2 & 2 & 2 & 4 & 9.00 & 0.000 \\
\hline
\end{tabular}

Input

The title, parameter, and data cards precede the data deck and are described in the comments at the beginning of the program listing. There is no program limitation for the number of variables (levels) which may be analyzed on each dimension. Also, there is no limit to the number of Ss used. However, there must be an equal number of Ss represented in each cell of the data matrix. As the program has no options for missing data, the user should either drop data from the analysis at random or should provide mean scores for the missing numbers before input of the data.

The data deck should be organized in blocks corresponding to rows, columns, and slices of a three-dimensional representation of the design. Figure 1 shows a representative deck structure. In all cases, including the four-dimensional design, the repeated measures factor is the basic input block index.

The raw input data must be numeric and in base 10 , but can be integer or explicit floating point.

\section{Output}

The following information is output from the program: (1) standard summary table, including variable names, mean squares, degrees of freedom, F ratios, and probability of each $F$ ratio; and (2) listing of mean and standard deviation for each cell (see Table 1).

\section{Error Detection}

The accuracy of output can best be determined by running a small deck of test data for which hand computations are available. Common sources of error involve keypunch errors in the source deck of the data, omissions of field-separating commas in the data, extra commas in the data, faulty parameter card, missing data, or incorrect input deck structure.

\section{PROGRAM LISTING}

ANOVA: PROCEDURE OPIIONS(MAIN);

/* THIS PROGRAM WILL PERFORM THE FOLLOWING ANALYSES

A. A 4 DIMENSIONAL TYPE III ANALYSIS OF VARIANCE COMPOSED OF 3 BETWEEN AND 1 WITHIN COMPARISON (TYPE 34 )

B. A 3 DIMENSIONAL TYPE III ANALYSIS OF VARIANCE COMPOSED OF 2 BETWEEN AND I WITHIN COMPARISON (TYPE 33).

C. A 2 DIMENSIONAL TYFE I ANALYSIS OF VAR IANCE COMPOSED OF 1 BETWEEN AND I WITHIN COMPARISON (TYPE O1)

IN ORDER TO USE THIS PROGRAM THE DATA CARDS MUST BE. IN THE FOLLOWING ORDER:

FIRST CAFD: HOLDS THE NAME OF THE EXPERIMENT. THIS NAME MAY

BE UP TO 40 SPACES LONG AND MUST BE ENCLOSED BY SINGLE QUOTES
AND FOLLOWED BY A COMA.

SECOND CARD: HOLDS THE TYPE AND DIMENSION OF THE ANALYSIS WHICH YOU WISH TO BE PERFORMED. THE TYPE COMES FIRST AND TS FOLLOWED BY A COMMA, THEN THE DIMENSION WHICH IS ALSO FOLLOWED BY A COMMA. THIRD CARD: HOLDS THE NAMES OF THE VARIABLES. EACH NAME MAY THE UP TO 10 SPACES IONG. THEY MUST BE ENCLOSED IN SINGLE QUOTES BE UF TO 10 SPACES IONG.
AND FOICOWED BY A COMMA.

FOURTH CARD: HOLDS THE NUMBER OF VARTABLES (LEVELS) THERE ARE FOF EACH DIMENSION. IN ADDITION THE NUMBER OF SUBJECTS PER CELL IS REQUIRED, EACH CELL HAVING EQUAL NUMBERS OF SUPJECTS. TELE COME FIRST, IN THAT ORDER, EACH FOLLOWED BY A COMMA. THEN THE NUMBER OF SURJECTS PER CELI FOLLOWED BY A COMMA. 
FIFTH - N CARDS: THESE ARE THE DATA CARDS UPON WHICH THE RAW SCORES ARE ENTERED. EACH SCORE MUST BE FOLLOWED BY A COMMA. THE SCORES SHOUID BE LISTED AS FOLLOWS: SCORES FOR CELL Al,Bl, Cl, D1, THEN FOR Al, Bl, Cl,D2, ETC. MORE THAN ONE CARD MAY BE USED FOR EACH CELLS SCORES IF NECESSARY.

EXAMPLE: THIS IS FOR A TYPE I ANALYSIS HAVING 2 VARTABLES ON THE BETWEEN DIMENSION AND 2 VARIABLES ON THE WITHIN DIMENSION WITH 5 SUBJECTS PER CELL.

CARD 1: 'EXAMPLE FOR TYYPE I'

CARD 2: 1,0 ,

CARD 3: 'BETWEEN ', 'WITHIN',

CARD 4: $2,2,5$,

CARD $5 \cdot-\mathrm{N}: 1,2,3,4,5$,

$6,7,8,9,10$

$11,12,13,14,15$

$16,17,18,19,20$

THE PROGRAM WILL NOW BEGIN *

DCL (TYPE, DIMEN , A , E , C , D , E , I , J , K , L , M , TN , N , DFT , DFBET, DFA , DFB, DFC , DFAB, DFAC, DFBC, DFABC, DFACD , DFBCD , DFABCD , DF ABD, DFEBET, DFWIN, $D F D, D F A D, D F B D, D F C D, F A, F B, F C, F D, F A B, F A C, F B C, F A B C, F A D, F B D$, FCD , FABD , FACD , FBCD , FABCD , X , Y Z Z, XX, YY , ZZ, XXX, YYY , ZZZ, DFEWIN, TSXS, TSX, CT , SST , STS , CB, SSBET , TVA, SSA, TVB , SSB, TVC, SSC, TAE, SSAB, TAC, SSAC, TBC , SSBC, SSERET , SSWIN, 'TDT , SSD , TAD , $\triangle S A D$, TBD , SSBD, TCD , SSCD, TABC, SSABC, TABD , SSABD, TACD, SSACD , TBCD , SSBCD, TABCD, SSABCD , SSEWIN, Q, $F$, MSA, MSB, MSC, MSD , MSAB, MSAC, MSAD , MSBC, MSBD MSCD , MSABC , MSABD, MSBCD , MSACD, MSABCD , MSEBET, MSEWT N, PROSA PROBB , PROBC, PROBAB, PROBAC, PROBEC, PROBABC , PROBD , PROBAD, PROBBD, PROBCD , FROBABD , PROBACD, FROHSCD , FKOBABCD \} DEC FLOAT INITIAL $(0)$;

DCL (VARA, VARB, VARC, VARD) CHALAITEF (10);

DCL PRBE ENTRY (DEC FLOAT, DEC FLOAT, DEC FINAT):

DCL (NAME) CHAR(40) VAPYING;

GET LIST(NAME);

GET IIIST (TYPE, DIMEN);

IF TYPE $=3$ THEN DO;

IF DIMEN $=4$ THEN 10 TO T3L;

END

IF DIMEN=3 THEN GO TO T33;

IF TYPE $=1$ THEN GO TO Tl;

T33: GET LIST (VARA, VARB, VARC):

$G E T \operatorname{LIST}(B, C, D, E)$

$A=1$;

GO TU START

T34: GET LIST (VARA, VARB, VARC, VARD);

$\operatorname{GET} \operatorname{LIST}(A, B, C, D, E)$

GO TO STAFT

TI: GET LIST (VARA, VARB);

$G E T \operatorname{LIST}(C, D, E)$;

$A=1$;

START:

$B=1$

BEGIN ;

$T N=\left(A^{*} B^{*} C^{*} D^{*} E\right)$;

$X=\left\{A^{*} B\right\}$

$Y=\left(A^{*} C\right)$

$\mathrm{Z}=\left(\mathrm{B}^{*} \mathrm{C}\right)$;

$X X=\left(A^{*} D\right)$

$Y Y=\left(B^{*} D\right)$

$\mathrm{ZZ}=\left(\mathrm{C}^{*} \mathrm{D}\right)$

$\mathrm{XXX}=\left(A * \mathrm{~B}^{*} \mathrm{D}\right)$;

$Y Y Y=\left(A^{*} C^{*} D\right)$

$Z Z Z=\left(B^{*} C^{*} D\right)$;

$\operatorname{DCL}(\operatorname{DATA}(A, B, C, D, E), D A Y T(A, B, C, D), G P T(A, B, C), S T(A, B, C, E), A A(A)$,

$B B(B), C C(C), A B(A, B), A C(A, C), B C(B, C), A D(A, D), B D(B, D), C D(C, D)$,

$A B D(A, B, D), A C D(A, C, D), B C D(B, C, D), D T(D), \operatorname{MEAN}(A, B, C, D), S D(A, B, C$,

D), $\operatorname{DAYSXS}(A, B, C, D), \operatorname{DAYTS}(A, B, C, D))$ DEC FLOAT

MEAN $=0 ; S D=0 ; D A Y S X S=0 ; D A Y T S=0 ; D A T A=0 ; D A Y T=0 ; G P T=0 ; S T=0 ; A A=0$;

$\mathrm{BB}=0 ; \mathrm{CC}=0 ; \mathrm{AB}=0 ; \mathrm{AC}=0 ; \mathrm{BC}=0 ; \mathrm{DT}=0 ; \mathrm{AD}=0 ; \mathrm{BD}=0 ; \mathrm{ABD}=0 ; \mathrm{BCD}=0 ; \mathrm{CD}=0 ; \mathrm{ACD}=0$ GET LIST (DATA)

1* COMPUTE DAY TOTALS, TOTAL SUM X SQ, DAY SUM $X$ SQ, TOTAL SUM $X$, GROUP TOTALS, DAY TOTALS SQ */

LI: DO I=I.TO A;

DO $\mathrm{J}=1$ TO $\mathrm{B}$;

$\mathrm{DO} \mathrm{K}=1$ TO $\mathrm{C}$;

DO $\mathrm{L}=1$ TO $\mathrm{D}$;

DO $M=\perp$ TO $E$;

$\operatorname{DAYT}(I, J, K, L)=\operatorname{DAYT}(I, J, K, I)+\operatorname{DATA}(I, J, K, L, M)$;

TSXS $=$ TSXS $+\left(\right.$ DATA $\left.(I, J, K, L, M) * *_{2}\right)$;

END

$\operatorname{DAYSXS}(I, J, K, L)=\operatorname{DAYSXS}(I, J, K, L)+(\operatorname{DATA}(I, J, K, L, M) * * 2)$;

$T S X=T S X+D A Y T(I, J, K, L)$

$\operatorname{GPT}(I, J, K)=G P T(I, J, K)+\operatorname{DAYT}(I, J, K, L)$

END WL:

$\operatorname{DAYTS}(I, J, K, L)=($ DAYT $(I, J, K, L) * 2) / F$;

/* COMPUTE CELl MEANS AND STD. DEV. */

WA: DO I=L TO A:

DO $\mathrm{J}=1$ TO $\mathrm{B}$

DO $\mathrm{K}=1$ TO $\mathrm{C}$;

DO $\mathrm{L}=1 \mathrm{TO} \mathrm{D}$;

$\operatorname{SD}(I, J, K, L)=S Q R T((\operatorname{DAYSXS}(I, J, K, I)-\operatorname{DAYTS}(I, J, K, L)) /(E-I))$

END LIA;

MEAN $(I, J, K, L)=D A Y T(T, J, K, L) / E$

Behav. Pes. Meth. \& Instru., 1971, Vol. 3 (4)
/* COMPUTE SUBJECT TOTALS, CORR. TERM, SS TOTAL */

L2: $D O \quad I=1$ TO A;

DO $\mathrm{J}=\mathrm{L}$ TO $\mathrm{B}$;

$\mathrm{DO} \mathrm{K}=\mathrm{I}$ TO $\mathrm{C}$;

DO $\mathrm{M}=1$ TO $\mathrm{E}$;

DO $\mathrm{L}=\mathrm{I}$ TO $\mathrm{D}$;

END Lê

$\operatorname{ST}(I, J, K, M)=\operatorname{ST}(I, J, K, M)+(D A T A(I, J, K, L, M))$;

$\mathrm{CT}=(\mathrm{TSX} * 2) / \mathrm{TN} ; \mathrm{SST}=\mathrm{TSXS}-\mathrm{CT}$;

/ COMPITE SS BETWEEN */

L3: DO $\mathrm{I}=1$ TO A;

DO $J=1$ TO $B$;

DO $\mathrm{K}=1$ TO $\mathrm{C}$;

DO $M=1$ TO $E$;

END L3;

STS $+(\operatorname{ST}(I, J, K, M) * 2)$

$\mathrm{CB}=\mathrm{STS} / \mathrm{D}$

SSBET $=C B-C T$;

IF TYPE $=\lambda$ TTHEN DO

$S S A=1$

$\operatorname{SS} B=1$

GO TO L8;

END;

* COMPUTE SSA */

L4: DO I=工 TO A;

DO $I=I$ TO $B$;

DO $K=1$ TO $C$;

$\mathrm{AA}(I)=\mathrm{AA}(I)+\mathrm{CPT}(I, J, K)$;

END L4

L5: DO $I=1$ IO A;

END $L 5$

TVA $=T V_{A}+\left((A A(I) * * 2) /\left(B^{*} C^{*} D^{*} E\right)\right)$;

IF TYPE $=3$ \& DIMEN=3 THEN SSA=1;

IF TYPE $=3$ \& DIMEN=4 THEN IDO;

END $S S A=T V A-C P$;

/* COMPUTE SSB */

LG: DO J=I TO B;

DO $\mathrm{I}=1 \mathrm{TO} \mathrm{A}$;

DO $K=1$ TO $C$;

$\mathrm{BB}(J)=\mathrm{BB}(J)+(\mathrm{GPT}(I, J, K))$;

END L6

L?: DO J=I TO $B$;

END L7,

VVB $=P P_{B}+\left(\left(B B(J)^{* * 2}\right) /\left(A^{*} C^{*} D^{*} E\right)\right) ;$

$\mathrm{SSB}=\mathrm{T} \mathrm{VB}-\mathrm{CT}$;

$l^{*}$ COMPUTE SSC */

L8: DO $K=1$ TO $C$;

DO I=I TO $A$;

DO $J=1$ TO $B$;

END $L 8$;

$\mathrm{CC}(\mathrm{K})=\mathrm{CC}(\mathrm{K})+(\operatorname{GPT}(\mathrm{I}, \mathrm{J}, \mathrm{K}))$;

L9: Do $K=1$ TO $C$;

$T V C=T V C+((C C(K) * * 2) /(A * B * D * E))$;

END LO.

$\mathrm{SSC}=\mathrm{rVC}-\mathrm{Cr}$;

$f^{*}$ compute SSAB *

L10: DO I=I TO A;

DO $\mathrm{J}=1$ TO $\mathrm{B}$;

DO $\mathrm{K}=1$ TO $\mathrm{C}$;

END;

$A B(I, J)=A B(I, J)+G E T(I, J, K)$;

END LIO

$S S A B=T A B-C T-S S A-S S B$

* compute SSAC */

L12: DO I=I TO A;

DO $K=1$ TO $C$;

DO $\mathrm{J}=1$ TO $B$;

$A C(I, K)=A C(I, K)+G P T(I, J, K)$;

END

$T A C=T A C+((A C(I, K) * * 2) /(B * D * E)) ;$

END Ll2;

$S S A C=T A C-C T-S S A-S S C$;

/* COMpur'e SSEC */

LI4: DC K=1 TO C;

DO $I=1$ TO $B$

DO $I=1$ TO $A$;

END;

$\mathrm{BC}(\mathrm{K}, \mathrm{J})=\mathrm{BC}(\mathrm{K}, \mathrm{J})+\mathrm{GPP}(\mathrm{I}, \mathrm{J}, \mathrm{K})$;

END LLL;

$S S B C=T B C-C T-S S B-S S C$ 
/* COMPUTE SSABC */

L6: DO I=I TO A;

DO $\mathrm{J}=1$ TO $\mathrm{B}$;

DO $\mathrm{K}=1$ TO $\mathrm{C}$;

$T A B C=T A B C+\left((\operatorname{GPT}(I, J, K) * * 2) /\left(D^{*} E\right)\right)$;

END 16

$S S A B C=T A B C-C T-S S A-\$ S B-S S C-S S A B-S S A C-S S B C$;

/* COMPUNE SS ERROR BETWEEN */

IF TYPE $=1$ THEN DO;

END;

SSEBET $=$ SSBET-SSC

IF TYPE $=3$ \& DIMEN=3 THEN DO

END; SSEBET $=$ SSBET-SSB-SSC-SSBC; END; SSEBET = SSBET-SSA-SSB-SSC-SSAB-SSAC-SSBC-SSABC;

$1 *$ COMPute ss WIThIN *

Ll7 : DO L=1 TO D;

DO $I=1$ TO $A$;

DO $\mathrm{J}=1$ TO $\mathrm{B}$;

DO $\mathrm{K}=1$ TO $\mathrm{C}$;

$\operatorname{DT}(\mathrm{L})=\mathrm{DT}(\mathrm{L})+\operatorname{DAYT}(\mathrm{I}, \mathrm{J}, \mathrm{K}, \mathrm{L})$;

DND $\mathrm{LI7}$;

L18: DO L=1 TO D;

$T D T=T D T+\left((D T(L) * 2) /\left(A * B^{*} C * E\right)\right) ;$

END L28;

$S S D=T D T-C T$;

\section{/* CONPUTE SSAD */}

I19: DO I=1 TO A;

$D O L=1$ TO $D$;

DO $\mathrm{J}=1$ TO $\mathrm{B}$;

DO- $K=1$ TO $C$;

$A D(I, L)=A D(I, L)+\operatorname{DAYT}(I, J, K, L)$;

END;

$T A D=T A D+\left((A D(I, L) * * 2) /\left(B^{*} C^{*} E\right)\right)$;

END 119

SSAD $=T A D-C T-S S A-S S D$;

/* COMPUTE SSBD *

L21: $D$ J $=1$ TO B;

DO $I=1$ TO $D$

DO $I=1$ TO A;

DO $K=1$ TO $C$

$B D(J, L)=B D(J, I)+D A Y T(I, J, K, I)$

BND;

$T B D=T B D+((B D(J, E) * * 2) /(A * C * E)\}$

END L21;

SSBD $=$ TBD-CT-SSB-SSD;

/* COMPUTE SSCD *

L23: DO K=1 TO C;

DO $L=1$ TO $D$

DO $J=1$ TO $B$

DO $I=I$ TO $A$

$\mathrm{CD}(\mathrm{K}, \mathrm{L})=\mathrm{CD}(\mathrm{K}, \mathrm{L})+\operatorname{DAYT}(\mathrm{I}, \mathrm{J}, \mathrm{K}, \mathrm{L})$

FiD;

$\mathrm{TCD}=\mathrm{TCD}+\left((\mathrm{CD}(K, L) * * 2) /\left(\mathrm{A}^{*} \mathrm{~B}^{*} \mathrm{E}\right)\right) ;$

END L23;

SSCD $=T C D-C T-S S C-S S D$;

/* COMPUTE SSABd */

L25: DO I=1 TO A

DO $\mathrm{J}=\mathrm{I}$ TO $\mathrm{B}$

DO $L=1$ TO $D$

DO $K=1$ TO C;

$A B D(I, J, L)=A B D(I, J, L)+D A Y T(I, J, K, L)$;

BRT;

END L25:

TABD $=T A B D+((A B D(I, J, L) * * 2) /(C * E))$;

SSABD-TABD-CT-SSAB-SSAD-SSBD-SSA-SSB-SSD;

* COMPUTE SSACD */

I2F: DO I=I TO A;

DO K=1 TO C;

DO $L=1$ TO D;

DO $J=1$ TO B;

$\operatorname{ACD}(I, K, L)=A C D(I, K, L)+\operatorname{DAYT}(1, J, K, L)$;

END;

END L2T

$A C D=\operatorname{MACD}+\left((A C D(I, K, L) * * 2) /\left(B^{*} E\right)\right) ;$

SSACD=TACD $-C T-S S A-S S C-S S D-S B A C-S S A D-S S C D$;
/* COMPUTĖ SSBCD *i

[29: DO $J=1$ TO $\mathrm{B}$

DO K=1 TO $\mathrm{C}$;

DO $L=1$ TO D

DO $I=\perp$ TO A

$\operatorname{BCD}(J, K, L)=\operatorname{BCD}(J, K, I)+\operatorname{DAYT}(I, J, K, L)$

END;

END L29;

$B C D=T B C D+\left((B C D(J, K, L) * 2) /\left(A^{*} E\right)\right)$

$\triangle S R C D=1 B C D-C T-S S B-S S C-S S D-S S B C-$ SSBD-SSCD;

* COMPUTE SSABCD */

L31: DO I=I TO A;

DO $J=1$ TO B;

DO $\mathrm{K}=1$ TO $\mathrm{C}$

DO $L=1$ TO $D$;

END L31

TABCD $=\mathrm{TABCD}+((D A Y T(I, J, K, L) * * 2) /(E))$

SSABCD=TABCD-CT-SSB-SSC-SSD-SSBC-SSAB-SSAC-SCADSSBD-SSCD-SSABC-SSABD-SSACD-SSBCD-SSA;

/* COMPUTE SS ERROR WITHIN */

IF TYPE=I THEN DO

END;

$$
\text { SSEWIN = SSWIN }- \text { SSD }- \text { SSCD; }
$$

END; SSEWIN =SSWIN - SSD - SSBD $-S S C D-$ SSBCD

IF TYPE $=3$ DIMEN $=4$ THEN DO; END; SSEWIN = SSWIN - SSD - SSAD $-S S B D-S S C D-S S A B D-S S A C D-S S B C D-S S A B C D$;

$1 *$ COMPUTE DF *

$\mathrm{DFT}=\mathrm{TW}-1$

DFBET $=\left(\left(A^{*} B^{*} C^{*} E\right)-1\right)$;

IF TYPE $=1$ THEN DO;

$\mathrm{DFA}=1$;

END;

IF TYPE $=3$ \& DIMEN $=3$ THEN DO; $D F A=1$;

END; $\mathrm{DFB}=\mathrm{B} \sim \mathrm{I}$;

TPE $=3$ \& DIMEN=4 THEN DO $\mathrm{DFA}=\mathrm{A}-\mathrm{\lambda}$;

END;

$\mathrm{DFB}=\mathrm{B}-1$;

$D F C=C-1$;

$D F A B=\left\{D F * *^{*} D E B\right\}$

$D F A C=\left(D F C^{*} D F A\right)$

$D F B C=\left(D F B^{*} D F C\right)$;

$D F A D C=(D F A * D F B * D F C)$;

IF TYPE=I THEN DO;

DFEBET=DFBET-DFC;

END;

IF TYPE $=3$ \& DIMEN $=3$ THEN DO

END; DFEBET =DFBET-DFB-DFC,DFBC

IF TYPE $=3$ \& DIMEN=4 THEN DO END; $D F E B E T=(D F B E T-D F A-D F B-D F C-D F A B-D F A C-D F B C-D F A B C)$;

$\mathrm{DFWIN}=\mathrm{DF}+\mathrm{DFBET}$

$D F D=D-1$

$D F A D=(D F A=D F D)$

$\mathrm{DFBD}=\left(\mathrm{DFB}{ }^{*} \mathrm{DFD}\right)$;

$\mathrm{DFCD}=(\mathrm{DFC} D \mathrm{DFD})$

$D F A B D=(D F A * D F B=D F D)$

$D F A C D=(D F A * D F C * D F D)$

$D F B C D=(D F B * D F C * D F D)$;

$\mathrm{DFABCD}=\left(\mathrm{DFA} \mathrm{DFB}^{*} \mathrm{UFC} \mathrm{FFO}^{*} \mathrm{DFD}\right)$

IF TYPE $=I$ THEN DO;

DFEW IN=DFWIN-DFD-DFCD;

END;

TF TYPE $=3$ \& $D$ LMEN $=3$ THEN DO

END DFEWIN=DFWIN-DFD-DFBD-DFCD-DFBCD; END;

DFEWIN=DFWIN-DFD-DFAD-DFBD-DFCD-DFABD-DFACD-DFBCD-DFABCD

I* coMpute MEAN SQ. *

MSA $=S S A / D F A$

$M S B=S S B / D F B$;

$M S C=S S C / D F C$

$\mathrm{MSD}=\mathrm{SSD} / \mathrm{DFD}$

$M S A B=S S A B / D F A B$

$M S A C=S S A C / D F A C$

$M S A D=S S A D / D F A D$

$M S B C=S S B C / D F B C$

$M S B D=S S B D / D F B D$

$M S C D=S S C D / D F C D$

$\mathrm{MSABC}=\mathrm{SSABC} / \mathrm{DFABC}$; 
$M S A B D=S S A B D / D F A B D$; $M S B C D=S S B C D / D F B C D$; $M S A C D=S S A C D / D F A C D$ $M S A B C D=S S A B C D / D F A B C D$; MSEBET $=$ SSEBET/DFEEET; MSEWIN=SSEWIN/DFEWII; $Q=$ MSEBET;

/* COMPUTE F RATIo */

$F A=M S A / Q$

$F B=M S B / Q$

$\mathrm{FC}=\mathrm{MSC} / \mathrm{Q}$

$F A B=M S A B / Q$;

$\mathrm{FAC}=\mathrm{MSAC} / \mathrm{Q}$

$F B C=M S B C / Q$;

$\mathrm{FABC}=\mathrm{MSABC} / \mathrm{Q}$;

$\mathrm{R}=\mathrm{MSEWIN}$

$F D=M S D / R$;

$F A D=M S A D / F$;

$F B D=M S B D / R$;

$F C D=M S C D / R$;

$\mathrm{FABD}=\mathrm{MSABD} / \mathrm{R}$;

$F A C D=M S A C D / R$

$\mathrm{FBCD}=\mathrm{MSBCD} / \mathrm{R}$

$F A B C D=M S A B C D / R$

/* COMPUTE PROBABILITIES */

IF TYPE= 1 THEN DO;

$P R O B C=P R B F(D F C, D F E B E T, F C)$;

$P R O B D=P R B P(D F D, D F E W I N, F D)$;

$P R O B C D=P R B F(D F C D, D F E W I N, F C D$ );

END GO TO P2;

IF TYPE=3 \& DIMEN=3 THEN GO TO F'L; PROBA $=$ PRBF $(D F A, D F E B E T, F A)$;

PROBAB=PRBF (DFAB, DFEBET, FAB );

PROBAC $=$ PRBF $($ DFAC, $D F E B E \Gamma, F A C)$

$P R O B A B C=P R B F(D F A B C, D F E B I T, F A B C)$

PROBAD $=$ PRBF $(D F A D, D F E W I N, F A D)$;

PROBABD $=P R B F(D F A B D, D F E W I N, F A B D)$;

PFOBACD $=$ PRBF (DFACD , DFEWIN, FACD);

PROBABCD =PRBF (DFABCD, DFEWIN, FABCD);

P1: $\quad$ PROBE $\triangle P R B F(D F B, D F E B E T, F B$ );

$P R O B C=P R B F(D F C, D F E B E T, F C)$;

$\mathrm{PROBD}=\mathrm{PRBF}$ (DFD, DFEWIN, FD);

PROBEC $=\mathrm{PRBF}$ (DFBC, DFEBET, FBC)

PROBED $=$ PRBF (DFBD, DFEWIN, FBD);

PROBCD $=$ PRBF (DFCD, DFEWIN, FCD);

PROBBCD=PRBF (DFBCD, DFENIN, FBCD);

P2: UNO $=$ NUO

F3: FORMAT $(X(6), A(5), X(9), 4 F(11,2), X(5), F(7,5))$;

F4: $\operatorname{FORMAT}(X(20), A(30))$;

F5: FORMAT $(X(10), A(6), X(10), A(2), X(10), A(2), X(8), A(2), X(10)$, $A(1), X(10), A(1)$

F6: FORMAT $(X(2), A(5), X(13), 2 F(11,2))$.

F7: $\operatorname{FORMAT}(X(4), A(16), 2 \mathrm{~F}(11,2))$;

F8: Formar $(X(6), A(1), X(1), A(1), A(10), A(1), 4 F(11,2), X(5)$, $F(7,5)$ );

F9: $\operatorname{FORMAT}(X(6), A(3), X(11), 4 F(11,2), X(5), F(7,5))$.

F10: FORMAT $(X(6), A(9), X(5), 3 F(11,2))$;

F11: FORMAT $(X(4), A(15), X(1), 2 F(11,2))$;

PUT PAGE;

IF TYPE $=1$ THEN GO TO TABLEl;

IF TYPE $=3$ THEN DO;

IF DIMEN $=4$ THEN GO TO TABLH 34

END;

IF DIMEN $=3$ THEN GO TO TABLE 33 ;

/* PRINT SUMMARY TABLES */

TABLE34

PUT EDIT (NAME) (X $(10), A)$;

PUT SKIP(2) EDIT('SUMMARY TABLE FOR TYPE 34 ANOVA') (R(F 4$)$ );

PUT SKIP (3):

PUT EDIT('SOURCE'', 'SS', 'DF', 'MS', 'F', 'P') (R(FS));

PUT SKIP (2):

PUT EDIT('TOTAL', SST, DFT) $(\mathrm{R}(\mathrm{F} 6))$;

PUT SKIP (1);

PUT EDIT('BETWEEN SUBJECTS', SSBE:,DFBET'; (R(F7))

PUT SKIP EDIT('A', '(', VARA,')', SSA, DFA, MSA, FA, PROBA) (R(F8));

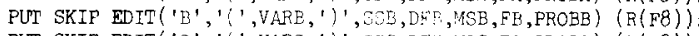
PUT SKIP EDIT(' 1 ', ' ', VARC,' ', SEC,OF',MSC,FC, $\mathrm{NROBC}$ ) (R(F8));

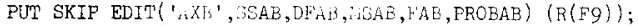
PUT SKIP EDIT ('AXC', SSAC, DFAC, MSAC, FAC, PROBAC) (R(F9)). PUT SKIF BDIT ('BXC', SSBC, DFBC, MSBC, FBC, PROBBC) (R(FO));

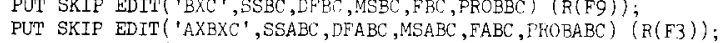
PUT SKIP EDIT( 'ERROR BET', SSEBET, DFEBET, MSEBET) (R(F1O)); PUT SKIP(2) EDIT('WITHIN SUEJECTS', SSWIN, DFWIN) (R(F11)); PUT SKIP EDIT ('D', '(',VARD, ')', SSD,DFD, MSD, FD, PROBD) (R(F8)); PUT SKIP EDIT ('AXD', SSAD, DFAD, MSAD, FAD, PROBAD) (R( 50$)$ ).

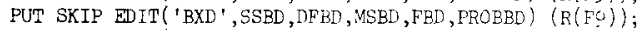
PUT SKIP EDIT('CXD', SSCD,DFCD,MSCD, FCD, PROBCD) (R(F9)); PUT SKIP EDIT( 'AXBXD', SSABD, DFABD , MSABD, FABD, PROBABD) (R(F3)); PU' SKIP EDIT ('AXCXD', SSACD, DFACD, MSACD, FACD, PROBACD) (R(F3));
PUT SKIP EDIT('BXCXD', SSBCD , DFBCD, MSBCD, FBCD, PROBBCD) (R(E3)) PITT SKIP EDIT( 'AXBXCXD' , SSABCD, DFABCD, MSABCD, FABCD, PROBABCD) $(X(6), A(7), X(7), 4 F(11,2), X(5), F(7,5))$

PUT SKIP EDTT ( $F R H O R$ WTN' SSEWIN, DFEWIN, MSEWIN) (R(F10));

PUT SKIP(S) EDIT('CELL MEANS AND STD DEV') $(X(10), A(22))$;

PUT SKIF(2) EDIT ('CELL', 'A', 'A' ', 'C', 'D', 'MEAN', 'STD DEV')

$(X(5), A(4), X(3), A(1), X(3), A(1), X(3), A(1), X(3), A(1), X(5), A(4)$,

$X(5), A(7))$;

DO $I=1$ TO A

DO $J=1$ TO B

DO $\mathrm{K}=1$ TO $\mathrm{C}$

DO $L=1$ TO $D$;

PUT SKIF EDIT(I, J,K,L,MEAN $(I, J, K, L), S D(I, J, K, L))$

$(X(1), F(3), X(1), F(3), X(1), F(3), X(1), F(3), X(3), F(6,2), X(3)$, $\mathrm{F}(7,3))$,

END;

END;

END;

TO STOP;

TABLE33:

PUT EDIT(NAME) $(X(20), A)$;

PUT SKIP(2) FDIT('SUMMARY TABLE FOR TYPE 33 ANOVA') (R(F4));

PUT SKIP(3) FOIT ('SOURCE', 'SS', 'DF', 'MS', 'F', 'P') (R(F5))

PUT SKIP(2) FDIT('TOTAL',SST,DFT) (R(F6));

PUT SKIF EDIT('BEIWEEN SUBJECTS', SSBET,DFBET) (R(F7));

PUT SKIP EDIT ('A' ' (', VARA, ')', SSB, DFB, MSB, FB, PROBB) (R(F8));

PUT SKIP EDIT ('B',' (', VARB,')', SSC, DFC, MSC, FC, PROBC) (R(F8));

PUT SKIP EDIT ('AXB', SSBC, DFBC, MSBC, FBC, PROBBC) (R(F9));

PUT SKIP EDIT ('ERROR BET', SSEBET, DFEBET, MSEBET) (R(F10));

PUT SKIP(2) EDIT('WIIHIN SUBJECTS', SSWIN, DFWIN) (R(F11));

PUT SKIP EDIT ('C' ' ' (', VARC, ')', SSD, DFD, MSD, FD, PROBD) (R(F8));

PUT SKIP EDIT ('AXC', SSBD, DFBD, MSBD, FBD, PROBBD) (R(F9));

PUT SKIP EDIT ('BXC' ', SSCD, DFCD, MSCD,FCD, PROBCD) (R(F9));

PUT SKIP EDIT ('AXBXC', SSBCD, DFBCD, MSBCD, FBCD, PROBBCD)

$(X(6), A(5), X(9), 4 F(1] .2) \cdot X(5), F(7,5))$ :

PUT SKIP EDIT ('ERROR WIN', SSEWIN, CFEWIN, MSEWIN) (R(FIO))

PUT SKIP(5) EDIT ('CELL MEANS AND STD DEV') $(X(10), A(22))$;

PUT SKIP(2) EDIT('CELL', 'A', 'B', 'C', 'MEAN', 'STD DEV')

$(X(5), A(4), X(3), A(1), X(3), A(1), X(3), A(1), X(5), A(4), X(5), A(7))$;

DO $I=1$ TO A;

DO $\mathrm{J}=1$ TO $\mathrm{B}$;

DO $\mathrm{K}=1$ TO $\mathrm{C}$

DO $L=1$ TO $D$

PUT SKIP EDIT( $J, K, L, M E A N(I, J, K, L), S D(I, J, K, L))$

$(X(11), F(3), X(1), F(3), X(1), F(3), X(3), F(6,2), X(3), F(7,3))$;

END;

END;

END;

GO TO STOP;

TABLE 1:

PUT EDIT (NAME) (X(10), A);

PUT SKIP(2) EDIT('SUMMARY TABLE FOR TYPE OI ANOVA') (R(F4));

PUT SKIP(3) EDIT ('SOURCE', 'SS', 'DF', 'MS', $\left.{ }^{\prime} F^{\prime},{ }^{\prime} P^{\prime}\right)$ (R (F5));

PUT SKIP(2) ENTT ' 'TOTAL', SST, DFT) (R(F6) );

PUT SKIP EOIT ('BETWEEN SUBJECTS' SSBET,DFBET) (R (F7))

PUT SKIP EDIT('A', '(', VARA, ')', SSC, DFC, MSC, FC, PROBC) (R(F8));

PUT SKIP EDIT ('ERROR BET', SSEBET, DFEBET, MSEBET) (R(F1O));

PUT SKIP(2) EDIT('WITHIN SUBJECTS', SSWIN, DFWIN) (R(FII));

PUT SKIP EDIT('B', '(', VARB, ')', SSD, DFD, MSD, FD, PROBD) (R(E8));

PUT SKIP EDIT ('AX8', SSCD, DFCD, MSCD, FCD, PROBCD) (R(F9));

PUT SKIP EDIT ('ERROR WIN', SSEWIN, DFEWIN, MSEWIN) (R(F10))

PUT SKIP(5) EDIT ('CELL MEANS AND STD DEV') $(X(10), A(22))$;

PUT SKIP(2) EDIT ('CELL', 'A', 'B', 'MEAN', 'STD DEV')

$(X(5), A(4), X(3), A(1), X(3), A(1), X(5), A(4), X(5), A(7))$

DO $L=1$ [O $A$;

DO $J=1$ TO B;

DO $K=1$ TO $C$;

DO $L=1$ TO $D$;

PUT SKIP EDIT(K, L, MEAN $(I, J, K, L), S D(I, J, K, L))$

$(X(11), F(3), X(1), F(3), X(3), F(6,2), X(3), F(7,3))$;

END;

END;

END;

END;

GO TO STOP

END;

TOP: PUT PAGE;

/ * PROBABILITY FUNCTION */

PRBF: PROCEDURE(DA, DE, FR) FLOAT

$\because$ DA=NUM DF, DB=DNOM DF, FR=F RAT1O $\%$

DCL(DA,DE, FR, PROB) DEC FLOAT;

$\mathrm{DCL}(A P, B P, F P, A A P, B B P, Z P)$ DEC FLOAT INITIAL(O); $P R O B=1.0$

IF ((DA*DB*FR)=0) THEN RETTRN (PROB);

IF FR< 1.0 THEN GO TO PIVE;

$\mathrm{AP}=\mathrm{DA}$;

$\mathrm{BP}=\mathrm{DB}$;

$\mathrm{FP}=\mathrm{FR}$;

GO TO TEN;

FIVE:

$\mathrm{AP}=\mathrm{DB}$;

$\mathrm{FP}=1.0 / \mathrm{FR}$; 


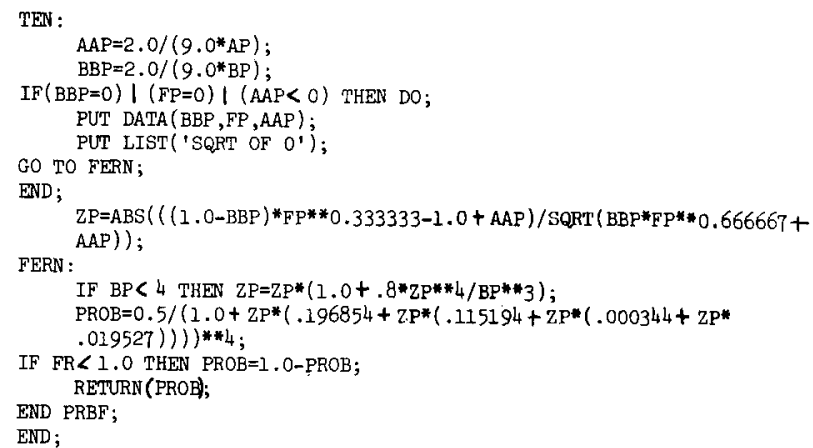

\title{
High-speed point plotting with computer-controlled displays*
}

\author{
L. WOJNAROWSKI, K. BACHMAN, and I. POLLACK † \\ University of Michigan, Ann Arbor, Michigan 41804
}

A method is described for the high-speed plotting of many independently generated elements in a computer-controlled display.

We shall describe a method of high-speed point plotting which we have found to be extremely useful. We are aware that special-purpose hardware-software display systems, e.g., character generators, vector plotters, are available to simplify certain display programs and to gain speed. The present method may be regarded as a supplement to, rather than a replacement of, such special-purpose display systems. The method may be expected to be useful when an extremely large number of independently generated points must be plotted within a short period of time.

The specific problem we faced was to paint four $32 \times 32$ element binary-coded displays upon a high-speed display scope (Tektronics 602 with P15 phosphor) without noticeable flicker. The two binary states-dot and no-dot-were equiprobable. We thus sought to plot the positions of approximately $0.5 \times 4 \times 32 \times 32$, or 2,048 , independently calculated dots. In order to avoid flicker upon successive paintings, we aimed to paint succcessive displays within 25 , and preferably within 20 , msec.

Several schemes are obvious candidates for consideration. Each may be the solution for a particular display system, depending upon the tradeoffs available for speed and for storage.

*The activities were supported, in part, by Grant $14036 \mathrm{X}$ of the National Science Foundation.

†Mailing address: Irwin Pollack, Mental Health Research Institute, 205 N. Forest Street, Ann Arbor, Michigan 48104 .
Scheme A is the most economical of machine storage, but is the slowest for displays with a large number of points. Displays are painted point by point as each point is calculated. No intermediate storage is required. This method of "on-line" plotting is excellent for displays with a very small number of display elements. The method is, however, hampered by the intermediate computational time required, especially for displays with a large number of display elements.

Schemes B and C are more costly of machine storage and are slower than Scheme A in terms of the delay from the beginning of computation to the display of the first point. The display cycle, however, is faster than for Scheme A, especially with a large number of display elements. Instead of computing and painting individual dots as in Scheme A, Schemes B and C first compute the binary state of each point and store the results of the successive computations. If the same display is presented over and over for successive paintings, the computation need be performed only once. In its most economical form for storage, Scheme B stores the binary code. The plotting routine scans the stored binary code, and in a plotting routine with a fixed geographical sequence, plots each binary element " 1 " and does not plot each binary element " $0 . "$ This system is relatively economical of storage since the binary coding is stored, and the individual words can be "unpacked" bit by bit. In our laboratories, Lon Radin has successfully used this method to plot four $60 \times 60$ element matrices with
BRUNING, J. L. \& KINTZ, B. L Computational handbook of statistics. Glenview, Ill: Scott, Foresman, 1968. Pp. 61-72.

LINDQUIST, E. F. Design and analysis of experiments in psychology and education. Boston: Houghton Mifflin, 1953. Pp. 281-284.

IIPPMAN， L. G., GALOSY， R. A., \& THOMPSON, R. W. Passive avoidance learning in gerbils and rats. Journal of Comparative \& Physiological Psychology. $1970,73,269-273$.

VELDM AN, D. J. Fortran programming for the behavioral sciences. New York: Holt, Rinehart \& Winston, 1967.

the PDP-8I computer (4,096 12-bit words). A storage display scope must be employed, however, because the conversion from binary code to geographical position takes too long for flickerless displays.

Scheme C, like Scheme B, first performs all of the necessary computations to determine the binary state of each display element. Scheme C carries the computations one step further. Instead of storing the binary code, Scheme C computes and stores the coordinates of each to-be-plotted point. Scheme $\mathrm{C}$ is more wasteful of storage than Scheme B, since the coordinate, rather than the binary state, information must be stored. On the other hand, because only the coordinates of the to-be-plotted locations are stored, the no-dot elements may be ignored. The resulting display routine can be made extremely tight.

In our first use of Scheme $\mathrm{C}$, we picked up the $x$ coordinate of the to-be-plotted point and instructed the $x$ digital-to-analog converter to generate and hold the respective $x$ voltage; we then picked up the $y$ coordinate of the to-be-plotted point and instructed the $y$ digital-to-analog converter to generate and hold the respective y voltage. Then we fired the $\mathrm{z}$ axis of the display scope. The steps were: fetch $x$, generate and hold $x$, fetch $y$, generate and hold $y$, fire $z$. The display cycle alone took 16 microsec, and, with housekeeping, took 22 microsec per point on the PDP-9 computer. The result was uns a tisfactorily long$2,048 \times 22$ microsec, or $44 \mathrm{msec}-$ for the displays we planned to use.

We had exhausted the three schemes without solving our initial goal and became discouraged. Then we got lucky. We noted that we were not using the full 12 bits of our digital-to-analog converters. We figured that we could get away with only 9 bits' resolution upon each coordinate, since plotting took place upon only 64 lines. We decided to store both the $\mathrm{x}$ and $\mathrm{y}$ coordinates of each point within a single word of the 